\title{
ON THE DESIGN OF INTERWORKING REFERENCE POINTS FOR INFORMATION NETWORKING
}

\section{Tim Eckardt, Christian Egelhaaf and Peter Schoo}

\author{
GMD FOKUS, \\ Kaiserin-Augusta-Allee 31, \\ D-10589 Berlin, Germany
}

eckardt@fokus.gmd.de, egelhaaf@fokus.gmd.de, schoo@fokus.gmd.de

\begin{abstract}
Providers of telecommunications services will have a need in the future to integrate a variety of applications into their service portfolios. They will make use of reference points, which allow interworking of applications in different administrative domains, like e.g. the Retailer Reference Point developed by TINA-C. Based on experiences with implementations that are compliant to such reference point specifications, the paper presents a new approach for a structured and modular composition of reference points. It allows eliminating overlap in functionality when combining multiple Business Relationships to be supported at one reference point between interacting administrative domains.
\end{abstract}

Keywords: TINA, reference point, conformance, segmentation, telecommunication, retailer, service provider, access session

\section{INTRODUCTION}

The TINA Consortium has developed a specialisation of the RM-ODP [1] applicable to the problem domain of telecommunications. This group of about 40 telecommunication companies and software vendors developed solutions that include suggestions for the development and the provisioning of services by means of distributed applications. The environment TINA has described enables stakeholders 
to provision services, each with capabilities provided in their own administrative domain. To achieve interworking across different administrative domains requires the existence of interworking reference points.

The roles in the TINA Business Model [3] are Service Providers, Retailers, Network Operators, End- users and Brokers, each being responsible for its administrative domain. The business model defines the relationship between different interworking roles, e.g. for the purpose of service provisioning. Based on this model TINA-C has identified a number of potential reference points [3], which allow interworking over domain boundaries and are candidates for conformance testing. A reference point (RP) which is completely defined at the moment is the Retailer Reference Point (Ret-RP). It supports the relationship between a consumer (end-user) and a retailer [4]. A retailer offers access to services and a consumer can make use of the provided services. More details are given below.

Reference Points enable parties in each of these roles to use applications that may come from any software provider. In the context of RM-ODP they are interworking reference points [Sec. 15.3.3, 2]. The conformance at these reference points can be tested by means of the interactions that are exchanged via the used communication protocol . According to the distributed responsibilities, conformance tests can increase the confidence in the distributed application for the interworking domains.

In a couple of projects $[5,6,7]$ the authors made experiences with the application of implementations that are compliant to the Ret-RP specification. One conclusion of these projects is that the monolithic Ret-RP could be improved to allow a more flexible reference point specification and consequently more efficient implementations. On the other hand, when it comes to the definition of other reference points identified by TINA, the results help to formulate a more sophisticated structuring for such reference points - a structure that

- builds on some of the properties of the existing Ret-RP,

- supports evolutionary developments, and thus allows for more viable reference point specifications and implementations, and which

- is applicable more widely.

Based on this motivation, the paper is structured as follows: First some background information regarding the TINA Retailer Reference Point and some specific TINA concepts are given, which are required for the understanding of this paper; next the approach for a structured and modular composition of reference points is presented, which is followed by an example; subsequently the paper is concluded.

\subsection{The TINA Retailer Reference Point}

TINA has defined a session as "the temporary relationship among a group of objects that are assigned to collectively fulfil a task for a period of time. A session has a state that may change during its lifetime. The session represents an abstract, simplified view of the management and usage of the objects and their shared information" [8]. It distinguishes access sessions, service sessions, and communication sessions. This 
separation allows the introduction of new services independently of the underlying network more easily. Access and service sessions are defined to work across multiple domains thus allowing retailers and third party service providers to offer compound services in a business environment.

The Ret-RP distinguishes an access part and an usage part. The operations defined in the access part allow to establish and release an access session, and to initiate the usage of a service. It contains the following functionality:

- initiation of trusted dialogue between the business administrative domains,

- identification of the business administrative domains to each other, note that either domain can remain anonymous dependent on the requested interaction,

- establishment of a secure association between the business administrative domains,

- set up of the default context for the control and management of service usage functionality,

- discovery of services being on offer,

- initiation of usage between the business administrative domains according to the agreed conditions,

- control and management of service sessions (e.g., stop, suspend, resume, join, notify changes, negotiate transfer of control rights, etc.).

The operations in the usage part are used within a service session, i.e. while using a service. The RP defines those interactions, which may be common to a number of different services:

- control and management of sessions, e.g., announce, stop, suspend, invite, notify changes, negotiate transfer of control rights,

- control and management of stream flow binding,

- business administrative domain management, e.g., subscriber management and service management.

The Ret-RP specification [4] encompasses information specifications, interface specifications and the observable behaviour (in terms of plain text) and is based on the TINA Business Model, which is a kind of enterprise model for the service provisioning in an environment encompassing a multitude of service providing retailers. The interface specifications are defined using OMG IDL. They describe the interfaces which are offered by the stakeholders to each other and which are located in different domains. An implementation conforms to the Ret-RP if it implements one or both of these parts correctly. 


\section{DESIGN GOALS AND PRINCIPLES FOR NEW REFERENCE POINTS}

Building on the concepts developed by TINA, this paper presents an approach for a structured and modular composition of upcoming reference points.

\subsection{General Goals}

The concept of Access Session as defined by the TINA Service Architecture [8] has to be generalised in a way to be applicable for any type of inter-domain reference point. Specifically, it should:

- enable the realisation of symmetric relationships which are independent of the party which initiated the access session;

- avoid the need for repeated authentication phases whenever a new context for interactions is to be established between two interacting domains already having established a secure and accountable association;

- allow to dynamically reconfigure interaction relationship which have already been set-up and instantiated.

A basic set of operations is required that should comprise interfaces supporting the management of such Generalised Access Sessions. Any additional functionality that has to be specified to support required business relationships across an interdomain reference point should be modularised into smaller, self-contained and re-usable components. For the ongoing work it is very important to build on the experience and specification efforts done for the TINA Ret RP, to avoid duplicated efforts and to protect existing assets.

\subsection{Conceptual Overview}

The general approach outlined in this paper is to structure inter-domain Reference Point into highly re-usable parts and defining a framework for the specification, application, and aggregation of these parts. The main motivation for the introduction of the concept of Reference Point Segments is to modularise the specification of reference points in a way allowing efficient combination of multiple Reference Point Segments according to the requirements arising from combined Business Relationships. The elementary concepts and their relationships are shown in Figure 1.

A reference point, which should support the interworking across domain borders, has to fulfil purpose-oriented interoperation requirements. The purpose is defined by a particular Business Relation. Different purposes within the Consumer/Retailer relationship are, for example, to start a service, to discover new services, etc.

Interfaces that enable interoperation are grouped by Reference Point Segments. As during an interoperation period several purposes can be addressed in the interworking, a reference point can comprise consequently multiple Reference Point Segments. By Reference Point Segments, operational contexts are established and necessary interfaces are identified. Vice versa, as the same purposes can be part of 
different Business Relationships, the aim is to identify Reference Point Segments meaningful to be used in other relationships too.

A necessary prerequisite, however, is, that the interoperation appears to be within a trusted relationship (Access Session) and the involved parties have agreed on the interworking purposes. This then is subject to the Generalised Access Segment.

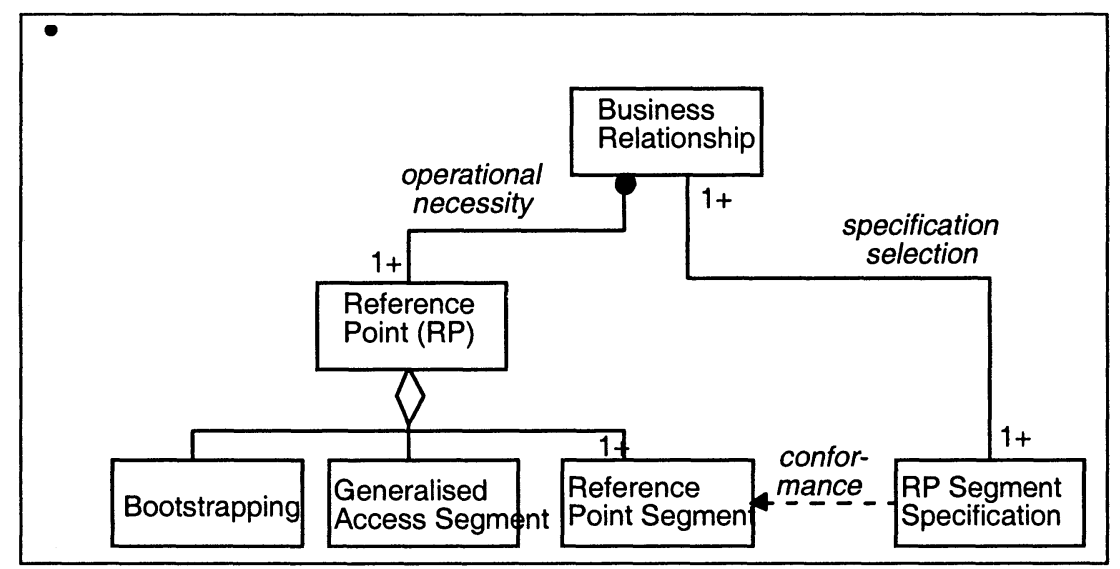

Figure 1. Relationship of the components forming a reference point, using OMT.

Shown in the centre of Figure 1, instantiated Reference Points between two administrative domains are structured as aggregations of re-usable components:

- a Generalised Access Segment which is a singleton needed independently of any particular Business Relationship to control and manage Access Sessions, including the launching of additional RP Segments;

- one or more Reference Point Segments which are fractions of interworking reference points dedicated to support particular Business Relationships; these segments will be launched by the functionality provided via the Generalised Access Segment;

- some standardised components for initiating a Reference Point between two domains, e.g. exchange of initial contact points (bootstrapping).

An aggregated Reference Point is needed between two separate administrative domains whenever a Business Relationship has to be supported in inter-domain interactions between two parties. The Business Relationships identified by the TINA Business Model, [3] serves as a basis for selecting those modules (i.e., Reference Point Segment Specifications) that are needed to support that particular Business Relationship. If multiple Business Relationships have to be supported in 
combination, individual segments needed for these Business Relationships can simply be re-used instead of having to be multiple instantiated.

All of these run-time components will be defined by dedicated specifications:

- a Base Reference Point specification defines the generic interface types to be instantiated as General Access Segments (not shown in figure 1);

- various Reference Point Segment Specification which define specific interface types according to the requirements imposed by the identified Business Relationship.

\section{FRAMEWORK FOR A GENERALIZED INTER DOMAIN REFERENCE POINT}

This section describes the main concepts comprising the framework for a generalised Interdomain reference point.

\subsection{Generalised Access Session}

According to the TINA Service Architecture, the concept Access Session is defined as a temporary relationship between two administrative domains allowing those domains to interact in a secure and accountable way. After the establishment of an Access Session, dedicated TINA nodes within both domains are enabled to send CORBA requests and receive replies or exceptions in a secure, controlled (and - if required - in a non- reputable way). Usually, this implies that each Access Session is associated with a dedicated security context where all participating parties are unambiguously identified and authenticated.

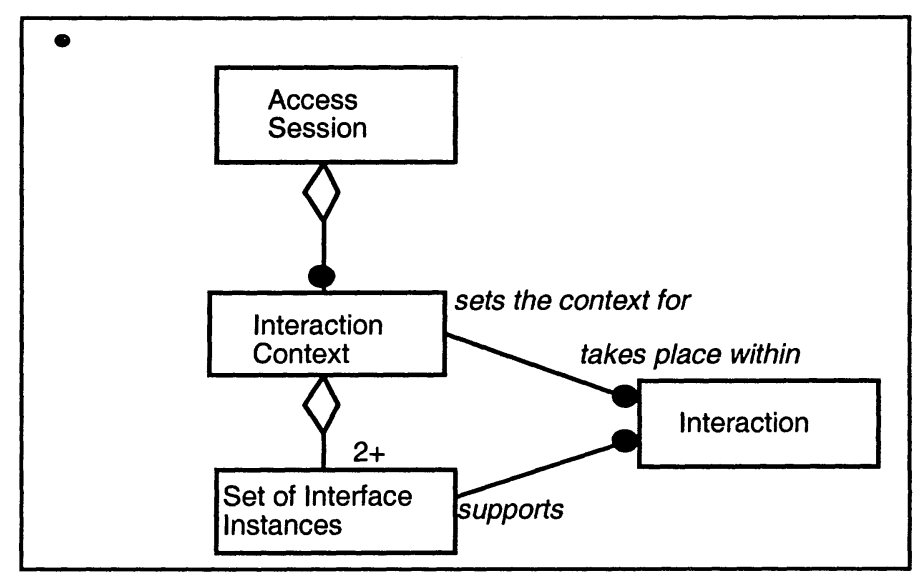

Figure 2. Run-time relationship between Access Session and Interaction Contexts. 
As a major enhancement compared with the Access Session concept defined by TINA, the Generalised Access Session has been modelled to be uniformly applicable to all Business Relationships defined by the TINA Business Model. Basically, this concept separates the aspects of authentication (and negotiation of the authentication methods) from the establishing of so-called Interaction Contexts, which includes the exchange of context-specific IORs within the Access Session. Special attention has been paid to the 'dynamics' of reference points (i.e., 'upgrading' of functionality during the lifetime of an Access Session as well as evolution of reference point specifications to future versions).

Interaction Contexts. Within an Access Session one or multiple Interaction Contexts can be established and maintained which associate related inter-domain interactions (as well as the interfaces supporting those interactions) during a certain period of time within the overall life time of the encompassing Access Session. Normally, there will be only one Interaction Context per Access Session. This concept has been introduced to provide a means for grouping and structuring related cross-reference point interactions within an Access Session as well as the interface instances supporting those interactions.

By separating the establishment of Interaction Contexts from the initial set-up of Access Sessions, a means for structuring interaction aspects is given. Any overhead associated with unnecessary re-authentication and other initial Access Session set-up procedures is avoided. The distinct mechanisms related to the set-up and management of Access Sessions become more generally applicable and flexible. The mechanisms defined for the establishment and control of Interaction Contexts are designed to offer flexibility in terms of evolution and dynamic adaptation of reference points. That is, at any time within an access session, additional Interaction Contexts can be established. A similar flexibility and adaptability holds for the Interaction Context itself.

Relation between Access Session and Interaction Contexts. There is a one-tomany relationship between an established Access Session and Interaction Contexts: an Access Session can 'hold' multiple separate Interaction Contexts but each individual Interaction Context exists only within exactly one Access Session. This Access Session can be symmetric or asymmetric. If both interacting domains are able to initiate and control Interaction Contexts, the Generalised Access Session is said to be symmetric. Otherwise, if only one party (this is usually the initiator of the Access Session) is able to control the life cycle of Interaction Contexts, the Access Session is asymmetric. Note, that the symmetry of an Access Session is not a question of mutual authentication: mutually authenticated domains can very well be involved in an asymmetric Access Session.

A single Interaction Context can comprise interactions related to more than one TINA Service Sessions, i.e., invitations exchanged between a service provider domain and a Retailer domain pertaining to different service sessions may use the same interface instances exchanged as part of the same Interaction Context.

Each Interaction Context is dynamically associated with a number of interface sets which may be enlarged or reduced during the life time of an Interaction Context: each party sharing the context supports a set of interfaces instances bound to this 
context for use by the peer domain(s). IORs for supported interfaces can be exchanged not only at the establishment of an Interaction Context but also at any time during its lifetime.

Usually, the set of interface types supported by one party are distinct from the other participant's sets. This may be due to the fact that they support asymmetric relationships such as the customer-provider relationship. Generally, within each Interaction Context, each participating party performs one or multiple precisely defined and well known role(s) which are mutually complementary. A scenario with identical sets of interface types would rather be the exception.

During its lifetime, each Access Session will evolve through a number of characteristic phases:

(i) set-up phase (identification and/or authentication takes place but has not been completed yet)

(ii) contexts establishing and modification phase (identification and/or authentication has been successfully completed; associated with this Access Session, an interface i_AssociationControl allowing to establish and control Interaction Contexts has been exchanged which is needed to establish and manage interaction contexts);

(iii) context usage phase (An Interaction Contexts has been established including the exchange of additional interfaces needed to support specific Business Relationships);

(iv) termination phase (the Access Session will be terminated; any interfaces pertaining to its remaining Interaction Contexts will be released)

An established Access Session can be unambiguously referred to by a dynamically assigned identifier, which will be exchanged during the set-up phase.

\subsection{Reference Point Segments}

All interfaces to be instantiated within a given Interaction Context for use "across a Reference Point" are grouped and packaged into Reference Point Segments. Thus a Reference Point Segment (denoting characteristic sets of interfaces) is the unit of reference point specification and instantiation.

Concerning the computational part, a Reference Point Segment is specified by a set of interface types of related functionality. For instance, functionality related to the charging or accounting business process could be grouped into a dedicated segment. A segment will be instantiated within a given Interaction Context as two groups of interfaces (for each of the interacting domains there is one group of interfaces to be supported by that domain; the remaining group in a Reference Point Segment is to be supported by the peer domain). Usually, both groups are not identical with respect to the contained interface types - only symmetric segments contain identical sets of interface types to be supported on both ends. The IORs to the interfaces supported by a domain have to be given to the peer domain, which is achieved with the help of operations being part of the Generalised Access Session segment. The number or types of Reference Point Segments, which have been 
instantiated within a given Interaction Context, may change during the life time of the Interaction Context.

\section{INTERFACES OF THE GENERALIZED ACCESS SEGMENT}

The specification of the Generalised Access Segment comprises specifications of three interface types:

\section{Interface i_DomainInitial}

RequestInitialAccess ()

Interface i_Domain Authenticate

GetAuthenticationMethods ()

authenticate ()

continueAuthentication ()

\author{
Interface i_AssociationControl \\ endAccess Session () \\ setupContext () \\ extendContext () \\ releaseContext () \\ releaseSegments ()
}

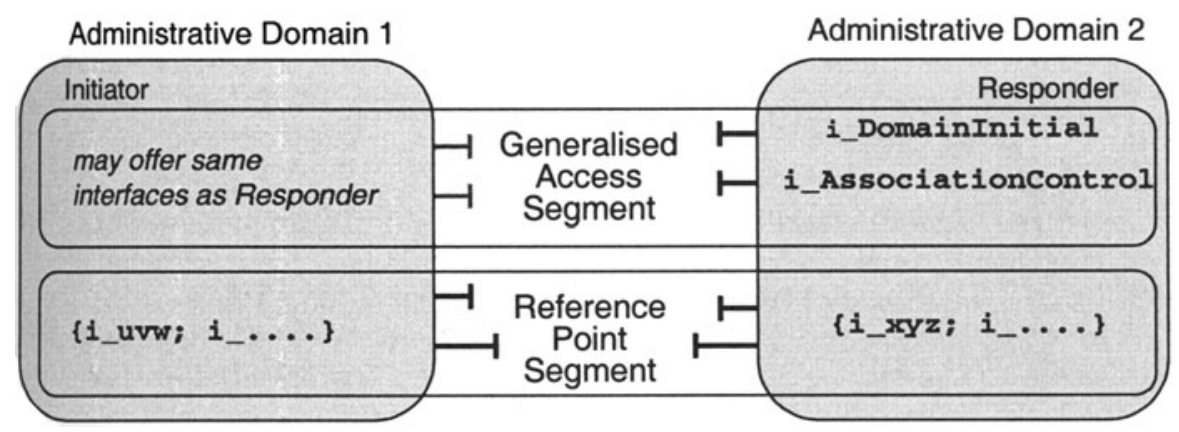

Figure 3. Initiator and Responder.

The relationship of these interfaces wrt. to administrative domains acting as Initiator and Responder of an Access Session is illustrated in Figure 3. Only interface instances (no computational objects supporting those interfaces) are shown, since the grouping of interface instances into supporting computational object is a design decision, which is left to the designers of the particular systems. The ability to interoperate does not depend on what grouping will be chosen.

The i_DomainInitial interface represents the initial contact point to the Responder domain. Unlike in the TINA specifications, the term 'Domain' is used instead of 'provider' or 'user' since any of the two, Responder or Initiator, could be the provider as well as the user. An IOR to this interface must be obtained in some way by the Initiator domain in order to establish an Access Session. The mechanism by which the Initiator domain gains a reference to this interface should be described as part of the bootstrapping prescriptions. The i_Domainlnitial interface is similar in functionality to i_Retailerlnitial defined for the Ret RP. The operation requestNamedAccess of the Ret reference point has been replaced by requestlnitialAccess providing similar but more generic and reusable functionality (i.e., identification and authentication of the responder/initiator as an alternative to authentication via ORB-level or transport-level mechanisms). 
After a successful authentication, the Responder will provide the Initiator with access to the i_AssociationControl interface, which can be used by the initiator during the life time of the Access Session to set-up, control and tear down Interaction Contexts within this Access Session. This interface allows to set-up and to negotiate any Interaction Contexts to be maintained within the Access Session and to manage those Interaction Contexts: it provides the mechanisms to add additional segments to an Interaction Context within an Access Session or to disable and delete segments from an Interaction Context established between two domains.

Finally, a dedicated operation provided at this interface allows to terminate the Access Session. This results in all Interaction Contexts including interfaces at both domains that have been created and exchanged for use within this particular Access Session will become invalid.

In the case only the initiator is able to access and use an i_AssociationControl interface, the Access Session is asymmetric. That is, only one of the two domains being involved in the Access Session is able to manage the life cycle of an access session and its comprised Interaction Contexts. An asymmetric Access Session can be upgraded to become a symmetric Access Session. This implies that both domains being involved in the Access Session support and offer an i_AssociationControl interface at their own domain for use by the peer domain during the life time of the Access Session.

The operation releaseContext is used to release all Reference Point Segments belonging to an existing Interaction Context. Usually, this implies to delete interfaces at both domains, the requester's domain as well as the responder's domain.

The operation releaseSegments is used to release the specified Reference Point Segments of the given Interaction Context and its comprised interfaces. Again, this would usually imply to delete interfaces at both domains, the Requester's domain as well as the responder's domain.

\section{CONCLUSIONS}

The paper presents an approach for the structuring and modular composition of interworking reference points in the sense of RM-ODP. For providers of telecommunications services this is considered important. In the future they will integrate a variety of applications into their service portfolios, and consequently will make use of interworking reference points. The presented approach allows to eliminate overlapping functionality when combining multiple Business Relationships to be supported at one reference point between two interacting administrative domains.

As result of the fine grained structuring into interworking purpose-oriented segments, which are selected on-line, the interworking efficiency is increased. It allows to handle more dynamic situations that may appear to be needed while the parties within a certain Business Relationship are interworking. This decreases operational overhead that would be required otherwise, since interaction contexts can be established and released on-line.

Besides the fine grained structuring the segmentation enables more evolutionary developments, this is useful for the augmentation of reference points to support changes required in the Business Relationships. This will lead to more handy reference point specifications, e.g. in case an additional interworking purpose is 
identified like the handling of invitations across the administrative domains of two retailers.

Smaller reference point portions would have been useful during the collaborative work that was driven by the TINA Core Team in the beginning of 1998, when the Ret-RP has been consolidated amongst a number of partners.

As it can be considered rather unrealistic that existing reference point specifications like the Ret-RP will be changed in the short term, there is nonetheless the opportunity to adopt the approach that is presented here for those reference point developments that are about to come in the near future. Consequently, the authors have introduced the concept of a generic access session to the TINA Working Group being responsible for the Service Architecture Reference Points (SARP) [9].

\section{References}

[1] ITU-T ISO/IEC Recommendation X.901 | International Standard 10746-1, Open Distributed Processing Reference Model: Overview, January 1995.

[2] ITU-T ISO/IEC Recommendation X.902 | International Standard 10746-2, Open Distributed Processing Reference Model: Foundations, September 1995.

[3] Mulder H. (Ed.), TINA Business Model and Reference Points, Ver 4.0, May 22, 1997

[4] Farley P., MinetTI R. (Eds.), TINA-C Ret Reference Point Specifications, Ver 1.0, 27 January 1998

[5] www.tinac.com/TTT/GlobalOne/globalone.html

[6] EgelhaAf C., Fisher M., Schoo P., Zisowsky A., Integration of a Legacy Application into a TINA Retailer Environment, ISADS 99, Tokyo March 21 -23 1999, accepted paper to be pubished

[7] www.eurescom.de/public/projects/P700-series/P715/p715pr.htm

[8] Kristiansen L. (Ed.), TINA-C Service Architecture, Ver. 5.0, 16 June, 1997

[9] www.tinac.com/TINA2000/workgroups/sarp.html

\section{Biographies}

Tim Eckardt, Christian Egelhaaf and Peter Schoo are working at GMD FOKUS since several years. FOKUS is a research institute of GMD located in Berlin, Germany. As senior scientists, the authors are involved in various research projects related to TINA-C. The projects are typically carried out for telecommunications industry and related consortia. 